\title{
Neurofeedback Rehabilitation Reduces Anxiety in Methamphetamine Abusers
}

\author{
Roghieh Nooripour ${ }^{*}$, Sverker Sikström², Nikzad Ghanbari3, Simin Hosseinian ${ }^{1}$, Peyman \\ Hassani-Abharian", and Hossein Ilanloo 5
}

${ }^{1}$ Department of Counseling, Alzahra University, Tehran, Iran

'Department of Psychology, Lund University, Lund, Sweden

${ }^{3}$ Clinical Psychology, Shahid Beheshti University (SBU), Tehran, Iran

${ }^{4}$ Institute for Cognitive Science Studies, Brain and Cognition Clinic, Tehran, Iran

${ }^{5}$ Kharazmi University, Tehran, Iran

\section{Abstract}

Addictive disorders are characterized by cognitive, behavioral, and neurological impairments caused by dysregulations of brain structure that can extend well beyond early withdrawal in the months and years of recovery. The present study aimed to examine the efficacy of neurofeedback rehabilitation on anxiety in methamphetamine abusers. The sample consisted of 14 male methamphetamine drug addicts who were randomly assigned to an experimental group $(n=7)$ and a control group $(n=7)$. Participants were assessed for Axis I disorders (SCID) and the Beck Anxiety Inventory (BAI). Mixed repeated ANOVA, independent $t$-tests, and chi-square were used for data analysis. The experimental group received 18 sessions of neurofeedback rehabilitation and standard psychological interventions treatment as usual, while the control group received only standard interventions. Results showed that neurofeedback significantly reduced anxiety in methamphetamine abusers at posttreatment and during a one-month follow-up. Along with other psychological interventions, neurofeedback rehabilitation is recommended for methamphetamine abusers.

Keywords: anxiety; methamphetamine abuse; neurofeedback; rehabilitation

Citation: Nooripour, R., Sikström, S., Ghanbari, N., Hosseinian, S., Hassani-Abharian, P., \& Ilanloo, H. (2021). Neurofeedback rehabilitation reduces anxiety in methamphetamine abusers. NeuroRegulation, 8(3), 128-136. https://doi.org/10.15540/nr.8.3.128

*Address correspondence to: Roghieh Nooripour, Department of Counseling, Alzahra University, North Sheikh Bahaee St., Dehe Vanak, Tehran, I. R. of Iran. P.Code: 1993893973. Email: r.noori@alzahra.ac.ir; nooripour.r@gmail.com

Copyright: (c) 2021. Nooripour et al. This is an Open Access article distributed under the terms of the Creative Commons Attribution License (CC-BY).

\section{Edited by:}

Rex L. Cannon, PhD, SPESA Research Institute, Knoxville, Tennessee, USA

\section{Reviewed by:}

Rex L. Cannon, PhD, SPESA Research Institute, Knoxville, Tennessee, USA

Mark Trullinger, The Chicago School of Professional Psychology, Washington, District of Columbia, USA; NeuroThrive, LLC, Lutherville, Maryland, USA

\section{Introduction}

Addictive disorders are characterized by cognitive, behavioral, and neurological impairments caused by dysregulations in brain structure that can persist well beyond early withdrawal through the months and years of recovery (Doostian et al., 2019). Research on psychological and neurophysiological dimensions of substance use disorders has recently attracted considerable attention (Mallorquí-Bagué et al., 2020; Richter et al., 2020). Patients suffering from substance use have been found to have a higher incidence of co-occurring Axis I and Axis II disorders, especially when stimulants are methamphetamine. Anxiety is the most common comorbid Axis I disorder in substance users. Anxiety disorder in young adults doubles the risk of later substance use (Albini et al., 2020). Emotional response levels at diagnosis of substance abuse are often lower with clinical comorbidity (Doostian et al., 2019). Self-medication in the form of amphetamine use occurs in patients with anxiety disorder, which is a risk for subsequent substance use. Psychological distress increases dropout rates during substance abuse treatment (Elkington et al., 2010; Roche et al., 2020). 
The early onset of mental illness, particularly anxiety, increases the likelihood of substance abuse. However, evidence suggests that pharmacotherapy in ADHD patients does not increase the risk of substance use disorder (Albini et al., 2020). Cooccurring anxiety disorders should be considered when treating substance abuse. Symptoms of methamphetamine dependence, such as craving, impulsivity, and psychological abnormalities, are associated with pathological neurophysiology (Fitzpatrick et al., 2020). Eugene Peniston first proposed treating addictive disorders with neurofeedback in 1991 (Imperatori et al., 2017). Research has shown that this method effectively reduces the psychological symptoms and effects of substance abuse (Askovic et al., 2020; Gruzelier et al., 2014; Kelley, 1997; Rostami \& Dehghani-Arani, 2015).

Neurofeedback based on electroencephalography (EEG) has recently been shown to be an efficient rehabilitation for substance abuse disorders (Nooripour et al., 2018). Feedback allows patients to change their rhythm and frequency of brain waves. Neurophysiological studies suggest that EEG provides information about the relationship between basic cortical brain mechanisms and psychological states in the field (Kosmyna \& Maes, 2019). Neurofeedback rehabilitation is a method that has recently been studied for its efficacy in psychopathological disorders such as stress and anxiety (Askovic et al., 2020; Harris et al., 2021; Hou et al., 2021). The method aims to help people change brainwave patterns without using invasive methods (Niv, 2013).

Neurofeedback rehabilitation is a way to condition the brain's electrical system, rewarding patterns that work well and inhibiting undesirable activities. It is also believed to stimulate growth and alter the efficiency of brain cells, improving brain function and cognitive-behavioral function (Davelaar, 2018). During neurofeedback rehabilitation, individuals learn to change the patterns of their brain waves through conditioning (Luctkar-Flude et al., 2017). Neurofeedback rehabilitation has been shown to promote relaxation, reduce stress, and alleviate psychological abnormalities in patients who are addicted to drugs and alcohol (Gray, 2017).

One method of neurofeedback rehabilitation is alpha/theta wave conditioning. Alpha brainwaves of frequency $8-12 \mathrm{~Hz}$ are associated with feelings of well-being (Gruzelier et al., 2014; Imperatori et al., 2017). Theta brainwaves of frequency $4-7 \mathrm{~Hz}$ have been associated with presleep state or daydreaming, including traumatic anxiety-provoking events with spontaneous hypnagogic imagery and/or abreaction (Gregory et al., 2020; Ticci et al., 2019). Alpha-theta rehabilitation is primarily designed to increase alpha and theta waves. Increasing the frequency of these waves enhances relaxation and the effects of conscious awareness (Imperatori et al., 2017). This relaxed state is referred to as the twilight state, in which the offender creates hypnagogic images or mental perceptions of repressed feelings and memories. These perceptions play a central role in the healing process. Few studies have examined neurofeedback rehabilitation for anxiety in substance abuse disorders (Chen \& Lin, 2020; Hammond, 2005; Simkin et al., 2014).

Hanslmayr et al. (2005) reported better performance in a cube rotation task after rehabilitation with upper alpha neurofeedback. Participants who were able to increase upper alpha amplitude (10-12 Hz) through neurofeedback rehabilitation showed better results in this task than participants who were unable to do so. Similarly, Vernon et al., (2003) reported that healthy participants were able to increase sensorimotor rhythm (SMR) activity after only eight neurofeedback rehabilitation sessions, and that this increase was associated with improvements in a cued recall task. Finally, Egner et al. (2004) trained participants to increase either SMR $(12-15 \mathrm{~Hz})$ or low beta rhythm $(15-18 \mathrm{~Hz})$. Previous studies recommended the SMR protocol as a training program through which participants could increase SMR and beta and downregulate theta (Avirame et al., 2016; Karageorghis et al., 2018). Increasing SMR to C4 (based on the international 10-20 system) is associated with a decrease in impulsivity symptoms and a facilitation of thalamic inhibitory mechanisms. In addition, increasing beta waves and decreasing excessive left hemisphere theta waves to $\mathrm{C} 3$ is recommended to improve responding. Although SMR training resulted in increased perceptual sensitivity, low beta training resulted in faster reaction times (Gruzelier, 2014).

Imaging techniques, such as SMR, functional magnetic resonance imaging (fMRI), and EEG, offer a window into the functioning brain, providing a unique opportunity to examine the neurobiological effects of these interventions in addiction. Imaging studies can be used to describe the brain systems involved in select interventions, clarify which mechanisms are dysfunctional, offering the opportunity to explore differences and commonalities between different interventions. Imaging-based neurobiological indicators entail information that goes beyond self-report or behavior 
alone and have been shown to be good predictors of relapse following treatment (Brewer et al., 2008; Janes et al., 2010; Moeller \& Goldstein, 2014). In SMR training protocol on the $\mathrm{Cz}$ area, the active electrode was placed at $\mathrm{Cz}$ with a left-ear reference (A1). The right earlobe was connected to circuit ground. In this program the reinforcement band was SMR $(12-15 \mathrm{~Hz})$ frequency band, and the suppressed frequency were delta $(2-5 \mathrm{~Hz})$, theta $(5-$ $8 \mathrm{~Hz}$ ) and high beta $(18-30 \mathrm{~Hz})$ frequency bands. Thresholds were adjusted in a way that if the participant maintained the reinforcement band above the threshold for $80 \%$ of the time during at least 0.5 $\mathrm{s}$, and the suppressed band under the threshold for $20 \%$ of the time, feedback was received. Whenever participants could maintain the reinforcement bands above the threshold for $90 \%$ of the time during two continuous trials, the threshold was changed automatically so that it was closer to the optimal threshold (Scott et al., 2005).

Due to the lack of sufficient research, neurofeedback rehabilitation is not yet recognized as a treatment for substance abuse problems; it is of interest to provide additional evidence of the efficacy of neurofeedback rehabilitation on anxiety in methamphetamine addicts.

\section{Method}

\section{Participants}

The population studied included a group of methamphetamine addicts in Tehran. Twenty-five participants who met the Diagnostic and Statistical Manual of Mental Disorders (5th ed.; DSM-5; American Psychiatric Association [APA], 2013) criteria for methamphetamine use disorder were selected. They were selected from Tehran drug rehabilitation centers between March 2019 and December 2019. Subjects were selected based on the following inclusion criteria:

1. They met DSM-V criteria for methamphetamine use disorder.

2. They showed interest in participating in the intervention.

3. They were between 20 and 50 years old.

4. They had at least a 12-month history of methamphetamine use.

5. They had no other substance-related disorders except smoking.

6. They could speak and write fluent Farsi.

The exclusion criteria were: Any history of psychiatric (bipolar disorder or major depression, psychosis) or neurological disorders.
Participants were randomly assigned to the neurofeedback rehabilitation group or the control group. Three subjects from the neurofeedback rehabilitation group and four from the control group dropped out before the study was completed. Therefore, the final analysis was performed on seven participants in the neurofeedback rehabilitation group $(n=7)$ and seven participants in the control group ( $n=7$; see Figure 1$)$.

\section{Procedure}

All participants were asked to complete the instruments (Structured Clinical Interview for DSM-IV [SCID] and Beck Anxiety Inventory [BAI]) before, at the midpoint, and after the intervention, and at a 4-week follow-up period. The neurofeedback rehabilitation group received the treatment protocol for 2.5 months. The protocol consisted of 18 sessions and two sessions per week, with each session lasting 25-30 minutes. The neurofeedback rehabilitation protocols in each session were based on SMR, $\mathrm{Cz}$ area (central cortex) rehabilitation (Scott et al., 2005), and Pz area (parietal cortex) alpha-theta (Gregory et al., 2020), performed in 20 min using the ProComp2 system (Thought Technology Ltd, Montreal, Canada; Scott et al., 2005).

\section{Measures}

Structured Clinical Interview for DSM-IV (SCID). The interview assessed the first Axis I Disorders in SCID-I. This included seven groups characterized by mood disorders as psychiatric issues, substance dependence, anxiety, eating complications, and compatibility problems. This instrument's reliability and validity are 0.81 to 0.84 , respectively (Sharifi et al., 2009).

Beck Anxiety Inventory (BAI). Beck Anxiety Inventory was used for rapid detection and differentiation from other disorders, particularly depression and anxiety (REF1988). This inventory assesses individuals' anxiety status during the last week on a 21-question multiple-choice self-report, where each answer is scored on a scale of 0 (not at all) to 3 (severely). Osman et al. (1997) reported a test-retest validity of 0.75 . Studies have indicated that this inventory's psychometric properties are desirable in Iran (Khesht-Masjedi et al., 2015).

Data Analysis. Data were analyzed by the mixed repeated ANOVA, independent $t$-test, and chisquare, using the statistical software SPSS-26. 
Figure 1. Summary of Patient's Flow Diagram.

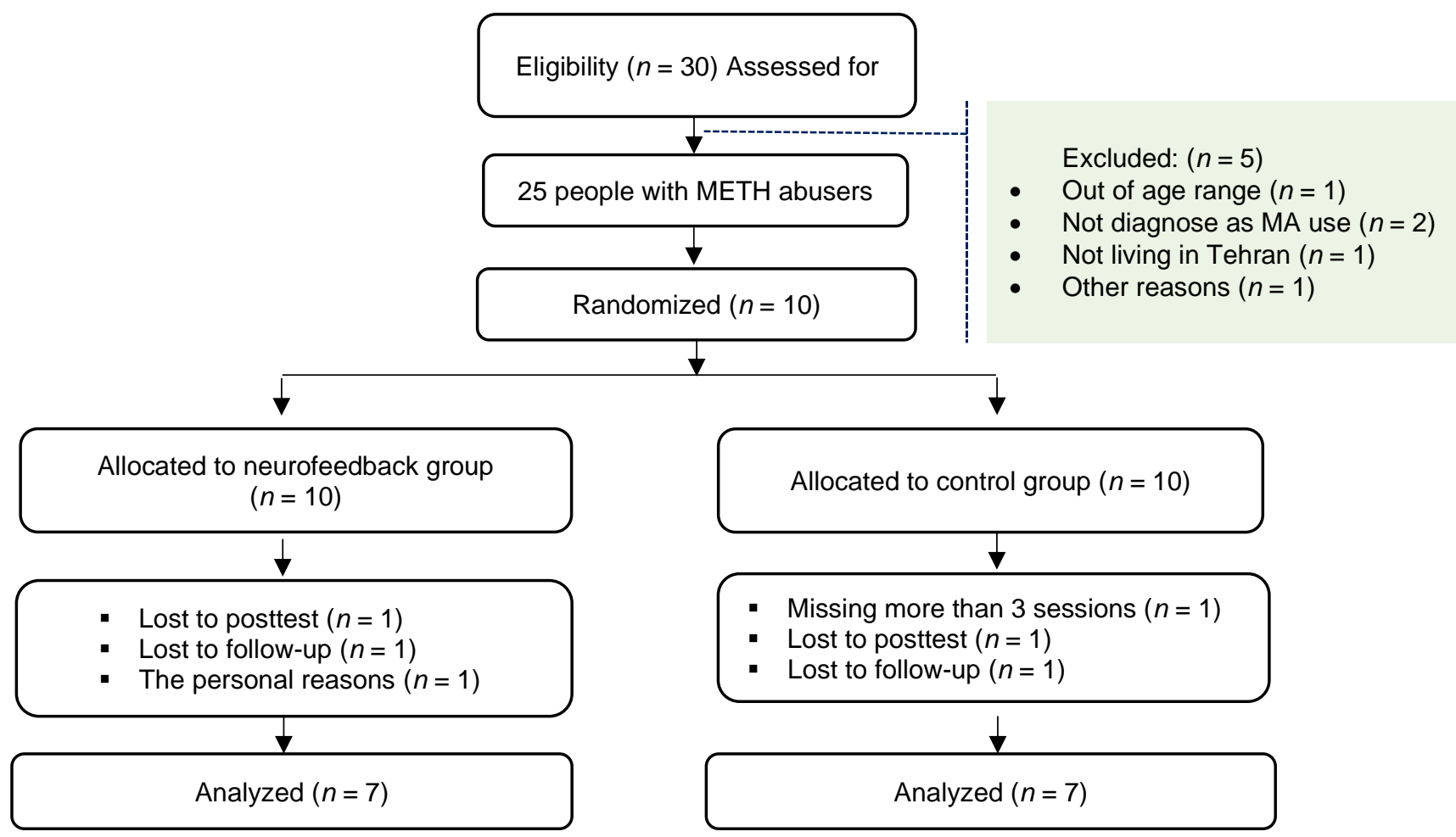

Ethical Consideration. The applied method is consistent with the National Research Committee's ethical standards, the Helsinki Declaration of 1964, subsequent revisions, or equivalent ethical norms. All participants in the study embraced informed consent to participate in the study.

\section{Results}

As for demographic variables, including age, marital status, job status, and substance use characteristics, there were no significant differences between the two groups (Table 1).

\section{Table 1}

Comparisons of Demographic Characteristics Across Groups

$$
\text { Neurofeedback }(n=7) \quad \text { Control }(n=7) \quad \text { Statistical Analyses }
$$

Job status (unemployed/part time/employed)

Marital status (single/married/divorced)

Age $(S D)$

Age of unset of substance use (years)

Methamphetamine abuse duration (years)
$37.14(4.56)$

18.82(4.06)

$3.23(1.43)$
$4.11(1.67)$

$$
\begin{aligned}
& X^{2}(2)=0.66, \text { n.s. } \\
& X^{2}(2)=0.47, \text { n.s. } \\
& t(12)=0.16, \text { n.s. } \\
& t(12)=0.23, \text { n.s. } \\
& t(12)=1.04, \text { n.s. }
\end{aligned}
$$


Repeated measures ANOVA was performed to test differences between the neurofeedback and control groups in anxiety. The group (neurofeedback vs. control) as between-subjects and the measurement time point (preintervention, midintervention 1-3, postintervention, and follow-up) as within-subjects were tested. There were no differences between the two groups on the pretest. There was a significant main effect between the neurofeedback and control groups, $F(1,12)=5.85, p \leq .05, \eta 2=.33$; a main effect of time (within-subject), $F(5,60)=25.8, p \leq$ $.001, \eta 2=.68$ (Table 2 and Figure 2); and an interaction effect of time (within-subject) with group (between-subjects) TIME $\times$ GROUP, $F(5,60)=$ 19.69, $p \leq .001, \eta 2=.62$.

\section{Table 2}

Summary of Beck Anxiety Inventory (BDI) Score in Pre-, Mid-, Postintervention, and 1-month Follow-up Measures and Mixed Repeated ANOVA

\begin{tabular}{cccccccccccc}
\hline Group & & T0 & T1 & T2 & T3 & T4 & T5 & Intervention & Time & Intervention*Time \\
Experiment & $M$ & 39.00 & 37.14 & 35.14 & 33.71 & 32.85 & 33.71 & $F=5.85$ & $F=25.80$ & $F=19.69$ \\
$(n=7)$ & $S D$ & 2.94 & 3.02 & 2.91 & 2.56 & 2.41 & 2.13 & $p \leq .05$ & $p \leq .001$ & $p \leq .001$ & \\
Control & $M$ & 38.28 & 38.28 & 38.14 & 37.85 & 38.14 & 37.71 & & & \\
$(n=7)$ & $S D$ & 2.28 & 1.88 & 1.77 & 1.67 & 2.03 & 1.79 & & \\
\hline
\end{tabular}

Note. $\mathrm{T} 0=$ preintervention $($ week 0$) ; \mathrm{T} 1=$ midterm $($ week 2$) ; \mathrm{T} 2=$ midterm $($ week 4$) ; \mathrm{T} 3=$ midterm (week 6); T4 = postintervention (week 8); T5 = 1-month follow-up (week 12).

Figure 2. Comparison of the Levels of Anxiety in Experimental (Neurofeedback) and Control Groups at Different Time Slots.

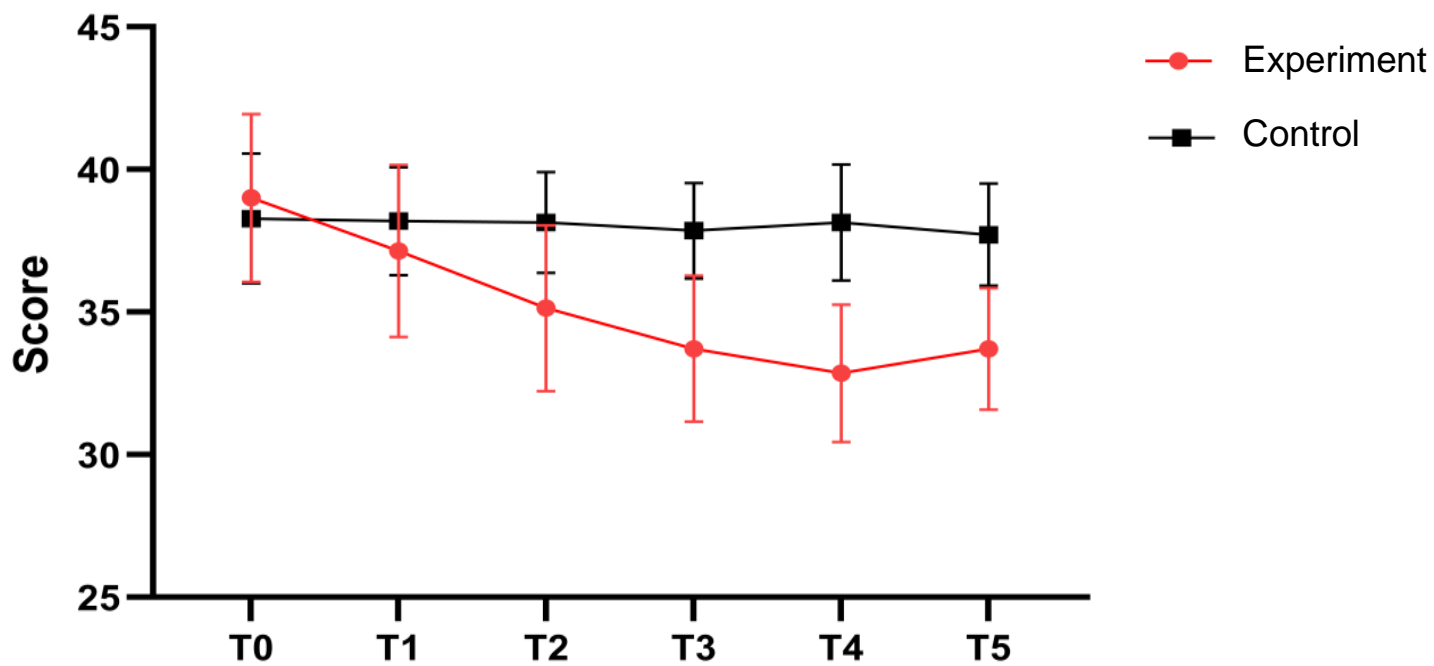

Assessment time points

Note. $\mathrm{T} 0$ = preintervention (week 0); T1 = midterm (week 2); T2 = midterm (week 4); T3 = midterm (week 6); $\mathrm{T} 4$ = postintervention (week 8); T5 = 1-month follow-up (week 12). 


\section{Discussion}

The current research examined the effectiveness of neurofeedback rehabilitation on anxiety in methamphetamine abusers. The results showed that neurofeedback rehabilitation significantly reduced anxiety among methamphetamine abusers during the posttreatment period. The same finding was also collected during a 1-month follow-up.

Neurofeedback rehabilitation is based on the idea that the mind can regenerate, change, and learn to heal itself. The mechanism associated with neurofeedback rehabilitation can be linked to a neurophysiological level. For example, a relationship between electroencephalograms and the thalamocortical mechanisms is responsible for the rhythms and frequencies of the electroencephalogram. Disrupted rhythms and frequencies of electroencephalograms can be normalized by neurofeedback rehabilitation, and these clinical effects are sustained (Niv, 2013). Our results on reduced anxiety are consistent with some studies, such as (Khajehpour et al., 2019; Liu et al., 2020).

Aliño Costa et al. (2017) found that EEG alpha had an anxiety-reducing effect on the experimental group. The use of an alpha-theta protocol in patients with anxiety disorder depends on the extent of the alpha area in the brain, especially in the parietal and occipital lobes. When the alpha range (8-12) is low, alpha enhancement may reduce anxiety symptoms. When the alpha range is high, a decrease in alpha can lead to anxiety-related symptoms. In the present study, the alpha enhancement protocol was used. According to Raymond et al. (2005), the alpha-theta protocol left the patient in theta mode.

Several studies, such as Gregory et al. (2020) have suggested that the most active and transformative properties of neurofeedback protocols teach substance abuse patients to intentionally intensify the amplitude and coherent interaction of their alpha and theta brainwave frequencies. The alpha-theta neurofeedback mechanism may allow participants to better tolerate stress, anxiety, and anxiety-provoking situations, especially during initial recovery periods.

Increased SMR amplitudes are associated with enhanced control of somatosensory and sensorimotor pathways, which could explain more accurate and even faster processing in reaction time paradigms. The basic idea is that individuals learn to self-regulate bioelectrical brain processes through operant conditioning, as measured by neurofeedback. Electrodes are placed on the scalp and specific parameters such as slow cortical potentials, alpha rhythm, SMR are extracted from the signal in real time. The participant is presented with easy to understand displays with simple shapes or video games. He is instructed to change the feedback display (e.g., increase or decrease the parameters of a bar or circle), which changes the associated brain activity. It would be interesting to conduct an SMR-NFT study that includes recording of neurofeedback during reaction time paradigms (Reichert et al., 2015). This would allow an analysis of SMR changes in relation to fast and slow responses, both within a session and across the entire experiment.

Schönenberg et al. (2017) also found no superiority of a theta/beta neurofeedback training over a metacognitive therapy or even a sham neurofeedback condition. Both (Bink et al., 2015; Schönenberg et al., 2017) applied the training in subjects with a single, well-defined disorder without any comorbidities. It is better the mechanism of SMR could be explored more. This pattern of results generally confirms that chronically hypoactive regions implicated in prefrontal control in drug addiction (Goldstein \& Volkow, 2011) can be normalized through cognitive and motivational/emotional interventions. Results also demonstrate that different cognitive interventions act, at least partly, through a common mechanism, supporting a previous meta-analysis that posited the recruitment of the inhibitory control network as a shared therapeutic mechanism between cognitive and pharmacological interventions (Konova et al., 2013).

Spectral amplitude estimates were calculated for the active site $(\mathrm{Cz})$ on raw 1-s EEG segments. A bandpass filter was used to extract the reward neurofeedback frequency band for SMR $(12-15 \mathrm{~Hz})$, and feedback was provided when the participant increased their SMR $(12-15 \mathrm{~Hz})$ by $10 \%$ for each baseline measured. Visual feedback was provided in the form of a graphic image animation. There was also a respiratory pacemaker with six cycles per minute, and the wave of heart rate and respiratory rate was measured to obtain physiological data from heart rate variability (HRV). This was done because the training of resonant frequency breathing affected the action of the somatic system (Vaschillo et al., 2006) and influenced the bottom-up circuit that produced SMR (Reid et al., 2013).

Drugs disrupted cognitive processes involving and affecting the hippocampal region and frontal cortex 
structures. Drugs have been shown to increase the process of apoptosis (planned cell death) and may exacerbate inhibition of neurogenesis (formation of neural tissue). One possibility is that neurofeedback reduces the cognitive deficits caused by substances by counteracting the apoptosis process and promoting neurogenesis. Limitations of the present study are using a self-report survey and the lack of a Sham or placebo group (fictitious stimulation). The SMR neurofeedback training can be considered as a training protocol to reduce anxiety in methamphetamine abusers. However, applying a cross-sectional design was a limitation to our study. Future studies are recommended to examine the obtained results through longitudinal designs. The results obtained suggest that therapists could use neurofeedback as an intervention method to treat methamphetamine addicts. Incorporating biological and neurological levels into future studies may be another step toward improving substance abuse treatment. Additionally, the specific significant changes during this neurofeedback protocol on neurological pathways can be studied with $\mathrm{fMRI}$ and PT scans in future studies.

\section{Author Contributions}

All authors contributed to the designing, conducting, and writing all parts of the research.

\section{Author Acknowledgment}

The authors extend their gratitude to all the clinics and participants for their cooperation with the project.

\section{Author Disclosure}

With an equal share of contribution to the project, the authors have no grants, financial interests, or conflicts to disclose.

\section{References}

Albini, M., Morano, A., Fattouch, J., Fanella, M., Avorio, F., Basili, L. M., Irelli, E. C., Manfredi, M., Giallonardo, A. T., \& Di Bonaventura, C. (2020). Anxiety as the only manifestation of focal non-convulsive status epilepticus: Case report and review of the literature. Neurocase, 26(2), 98-102. https://doi.org/10.1080/13554794.2020.1741647

Aliño Costa, M., Gadea, M., Hidalgo, V., Pérez, V., \& Sanjuán, J. (2017). An effective neurofeedback training, with cortisol correlates, in a clinical case of anxiety. Universitas Psychologica, 15(5). https://doi.org/10.11144 /Javeriana.upsy15-5.entc

American Psychiatric Association. (2013). Diagnostic and statistical manual of mental disorders (5th ed.). https://doi.org/10.1176/appi.books.9780890425596

Askovic, M., Watters, A. J., Coello, M., Aroche, J., Harris, A. W. F., \& Kropotov, J. (2020). Evaluation of neurofeedback for posttraumatic stress disorder related to refugee experiences using self-report and cognitive ERP measures. Clinical EEG and Neuroscience, 51(2), 79-86. https://doi.org/10.1177 1550059419849170

Avirame, K., Nuss, L., \& Todder, D. (2016). Safety and feasibility of neurofeedback training (NFB) during sleep in uncooperative child with autism: Case report. NeuroRegulation, 3(3), 135-139. https://doi.org/10.15540 /nr.3.3.135

Bink, M., van Nieuwenhuizen, C., Popma, A., Bongers, I. L., \& van Boxtel, G. J. M. (2015). Behavioral effects of neurofeedback in adolescents with ADHD: A randomized controlled trial. European Child \& Adolescent Psychiatry, 24(9), 1035-1048. https://doi.org/10.1007/s00787-014-0655-3

Brewer, J. A., Worhunsky, P. D., Carroll, K. M., Rounsaville, B. J., \& Potenza, M. N. (2008). Pretreatment brain activation during Stroop task is associated with outcomes in cocainedependent patients. Biological Psychiatry, 64(11), 998-1004. https://doi.org/10.1016/j.biopsych.2008.05.024

Chen, T.-C., \& Lin, I.-M. (2020). The learning effects and curves during high beta down-training neurofeedback for patients with major depressive disorder. Journal of Affective Disorders, 266, 235-242. https://doi.org/10.1016 /j.jad.2020.01.175

Davelaar, E. J. (2018). Mechanisms of neurofeedback: A computation-theoretic approach. Neuroscience, 378, 175188. https://doi.org/10.1016/j.neuroscience.2017.05.052

Doostian, Y., Bahmani, B., Farhoudian, A., Azkhosh, M., \& Khanjani, M. S. (2019). Vocational rehabilitation for individuals with substance-related disorders. Iranian Rehabilitation Journal, 17(2), 105-112. https://doi.org /10.32598/irj.17.2.105

Egner, T., Zech, T. F., \& Gruzelier, J. H. (2004). The effects of neurofeedback training on the spectral topography of the electroencephalogram. Clinical Neurophysiology, 115(11), 2452-2460. https://doi.org/10.1016/j.clinph.2004.05.033

Elkington, K. S., Bauermeister, J. A., \& Zimmerman, M. A. (2010). Psychological distress, substance use, and HIV/STI risk behaviors among youth. Journal of Youth and Adolescence, 39(5), 514-527. https://doi.org/10.1007/s10964-010-9524-7

Fitzpatrick, R. E., Rubenis, A. J., Lubman, D. I., \& Verdejo-Garcia, A. (2020). Cognitive deficits in methamphetamine addiction: Independent contributions of dependence and intelligence. Drug and Alcohol Dependence, 209, 107891. https://doi.org/10.1016/j.drugalcdep.2020.107891

Goldstein, R. Z., \& Volkow, N. D. (2011). Dysfunction of the prefrontal cortex in addiction: Neuroimaging findings and clinical implications. Nature Reviews Neuroscience, 12(11), 652-669. https://doi.org/10.1038/nrn3119

Gray, S. N. (2017). An overview of the use of neurofeedback biofeedback for the treatment of symptoms of traumatic brain injury in military and civilian populations. Medical Acupuncture, 29(4), 215-219. https://doi.org/10.1089 lacu.2017.1220

Gregory, J. C., Romero, D., \& Jones, M. S. (2020). Predictors of neurofeedback outcomes following aEEG individualized protocols for anxiety. NeuroRegulation, 7(1), 18-25. https://doi.org/10.15540/nr.7.1.18

Gruzelier, J. H. (2014). Differential effects on mood of 12-15 (SMR) and 15-18 (beta1) $\mathrm{Hz}$ neurofeedback. International Journal of Psychophysiology, 93(1), 112-115. https://doi.org /10.1016/j.ijpsycho.2012.11.007

Gruzelier, J. H., Foks, M., Steffert, T., Chen, M. J.-L., \& Ros, T. (2014). Beneficial outcome from EEG-neurofeedback on creative music performance, attention and well-being in school children. Biological Psychology, 95, 86-95. https://doi.org/10.1016/j.biopsycho.2013.04.005

Hammond, D. C. (2005). Neurofeedback with anxiety and affective disorders. Child and Adolescent Psychiatric Clinics of North America, 14(1), 105-123. https://doi.org/10.1016 /j.chc.2004.07.008 
HansImayr, S., Sauseng, P., Doppelmayr, M., Schabus, M., \& Klimesch, W. (2005). Increasing individual upper alpha power by neurofeedback improves cognitive performance in human subjects. Applied Psychophysiology and Biofeedback, 30(1), 1-10. https://doi.org/10.1007/s10484-005-2169-8

Harris, S., Hundley, G., \& Lambie, G. (2021). The effects of neurofeedback on depression, anxiety, and academic selfefficacy. Journal of College Student Psychotherapy, 35(1), 15-29. https://doi.org/10.1080/87568225.2019.1606689

Hou, Y., Zhang, S., Li, N., Huang, Z., Wang, L., \& Wang, Y. (2021). Neurofeedback training improves anxiety trait and depressive symptom in GAD. Brain and Behavior, 11(3), e02024. https://doi.org/10.1002/brb3.2024

Imperatori, C., Valenti, E. M., Della Marca, G., Amoroso, N., Massullo, C., Carbone, G. A., Maestoso, G., Quintiliani, M. I., Contardi, A., \& Farina, B. (2017). Coping food craving with neurofeedback. Evaluation of the usefulness of alpha/theta training in a non-clinical sample. International Journal of Psychophysiology, 112, 89-97. https://doi.org/10.1016 /j.ijpsycho.2016.11.010

Janes, A. C., Pizzagalli, D. A., Richardt, S., Frederick, B. deB., Chuzi, S., Pachas, G., Culhane, M. A., Holmes, A. J., Fava, M., Evins, A. E., \& Kaufman, M. J. (2010). Brain reactivity to smoking cues prior to smoking cessation predicts ability to maintain tobacco abstinence. Biological Psychiatry, 67(8), 722-729. https://doi.org/10.1016/j.biopsych.2009.12.034

Karageorghis, C. I., Bigliassi, M., Guérin, S. M. R., \& DelevoyeTurrell, Y. (2018). Brain mechanisms that underlie music interventions in the exercise domain. In S. Marcora \& M. Sarkar (Eds.), Progress in Brain Research (vol. 240, pp. 109125). Elsevier. https://doi.org/10.1016/bs.pbr.2018.09.004

Kelley, M. J. (1997). "Native Americans, neurofeedback, and substance abuse theory". Three year outcome of alpha/theta neurofeedback training in the treatment of problem drinking among Dine' (Navajo) people. Journal of Neurotherapy, 2(3), 24-60. https://doi.org/10.1300/J184v02n03_03

Khajehpour, H., Mohagheghian, F., Ekhtiari, $\bar{H}_{\text {., Makkiabadi, B., }}$ Jafari, A. H., Eqlimi, E., \& Harirchian, M. H. (2019). Computer-aided classifying and characterizing of methamphetamine use disorder using resting-state EEG. Cognitive Neurodynamics, 13(6), 519-530. https://doi.org /10.1007/s11571-019-09550-z

Khesht-Masjedi, M. F., Omar, Z., \& Kafi Masoleh, S. M. (2015). Psychometrics properties of the Persian version of Beck Anxiety Inventory in North of Iranian adolescents. International Journal of Educational and Psychological Researches, 1(2), 145-153. https://doi.org/10.4103/23952296.152233

Konova, A. B., Moeller, S. J., \& Goldstein, R. Z. (2013). Common and distinct neural targets of treatment: Changing brain function in substance addiction. Neuroscience \& Biobehavioral Reviews, 37(10 Part 2), 2806-2817. https://doi.org/10.1016/j.neubiorev.2013.10.002

Kosmyna, N., \& Maes, P. (2019). AttentivU: An EEG-based closed-loop biofeedback system for real-time monitoring and improvement of engagement for personalized learning. Sensors, 19(23), 5200. https://doi.org/10.3390/s19235200

Liu, W., Chen, X.-J., Wen, Y.-T., Winkler, M. H., Paul, P., He, Y.-L., Wang, L., Chen, H.-X., \& Li, Y.-H. (2020). Memory retrieval-extinction combined with virtual reality reducing drug craving for methamphetamine: Study protocol for a randomized controlled trial. Frontiers in Psychiatry, 11, 322. https://doi.org/10.3389/fpsyt.2020.00322

Luctkar-Flude, M., Groll, D., \& Tyerman, J. (2017). Using neurofeedback to manage long-term symptoms in cancer survivors: Results of a survey of neurofeedback providers. European Journal of Integrative Medicine, 12, 172-176. https://doi.org/10.1016/j.eujim.2017.06.003

Mallorquí-Bagué, N., Lozano-Madrid, M., Testa, G., VintróAlcaraz, C., Sánchez, I., Riesco, N., Perales, J. C., Navas, J.
F., Martínez-Zalacaín, I., Megías, A., Granero, R., Veciana de las Heras, M., Chami, R., Jiménez-Murcia, S., FernándezFormoso, J. A., Treasure, J., \& Fernández-Aranda, F. (2020). Clinical and Neurophysiological Correlates of Emotion and Food Craving Regulation in Patients with Anorexia Nervosa. Journal of Clinical Medicine, 9(4), 960. https://doi.org $/ 10.3390 / j c m 9040960$

Moeller, S. J., \& Goldstein, R. Z. (2014). Impaired self-awareness in human addiction: Deficient attribution of personal relevance. Trends in Cognitive Sciences, 18(12), 635-641. https://doi.org/10.1016/j.tics.2014.09.003

Niv, S. (2013). Clinical efficacy and potential mechanisms of neurofeedback. Personality and Individual Differences, 54(6), 676-686. https://doi.org/10.1016/j.paid.2012.11.037

Nooripour, R., Hosseinian, S., Afrouz, G. A., \& Bakhshani, N.-M. (2018). Effectiveness of neurofeedback on executive functions and tendency toward high-risk behaviors in adolescents with attention deficit hyperactivity disorder. International Journal of High Risk Behaviors and Addiction, 7(4), e82012. https://doi.org/10.5812/ijhrba.82012

Osman, A., Kopper, B. A., Barrios, F. X., Osman, J. R., \& Wade, T. (1997). The Beck Anxiety Inventory: Reexamination of factor structure and psychometric properties. Journal of Clinical Psychology, 53(1), 7-14. https://doi.org/10.1002 /(SICl)1097-4679(199701)53:1<7::AID-JCLP2>3.0.CO;2-S

Raymond, J., Varney, C., Parkinson, L. A., \& Gruzelier, J. H. (2005). The effects of alpha/theta neurofeedback on personality and mood. Cognitive Brain Research, 23(2-3), 287-292. https://doi.org/10.1016/j.cogbrainres.2004.10.023

Reichert, J. L., Kober, S. E., Neuper, C., \& Wood, G. (2015). Resting-state sensorimotor rhythm (SMR) power predicts the ability to up-regulate SMR in an EEG-instrumental conditioning paradigm. Clinical Neurophysiology, 126(11), 2068-2077. https://doi.org/10.1016/j.clinph.2014.09.032

Reid, A., Nihon, S., Thompson, L., \& Thompson, M. (2013). The effects of heart rate variability training on sensorimotor rhythm: A pilot study. Journal of Neurotherapy, 17(1), 43-48. https://doi.org/10.1080/10874208.2013.759020

Richter, F., García, A. M., Rodriguez Arriagada, N., Yoris, A., Birba, A., Huepe, D., Zimmer, H., Ibáñez, A., \& Sedeño, L. (2020). Behavioral and neurophysiological signatures of interoceptive enhancements following vagus nerve stimulation. Human Brain Mapping, 42(5) 1227-1242. https://doi.org/10.1002/hbm.25288

Roche, K., Moss, S., Baxendale, E., \& Stephens, S. (2020). Are psychological distress, substance misuse, and sexual sensation seeking associated with autoerotic asphyxiationrelated distress? The Canadian Journal of Human Sexuality, 29(2), 221-227. https://doi.org/10.3138/cjhs.2020-0004

Rostami, R., \& Dehghani-Arani, F. (2015). Neurofeedback training as a new method in treatment of crystal methamphetamine dependent patients: A preliminary study. Applied Psychophysiology and Biofeedback, 40(3), 151-161. https://doi.org/10.1007/s10484-015-9281-1

Schönenberg, M., Wiedemann, E., Schneidt, A., Scheeff, J., Logemann, A., Keune, P. M., \& Hautzinger, M. (2017). Neurofeedback, sham neurofeedback, and cognitivebehavioural group therapy in adults with attention-deficit hyperactivity disorder: A triple-blind, randomised, controlled trial. The Lancet Psychiatry, 4(9), 673-684. https://doi.org /10.1016/S2215-0366(17)30291-2

Scott, W. C., Kaiser, D., Othmer, S., \& Sideroff, S. I. (2005). Effects of an EEG biofeedback protocol on a mixed substance abusing population. The American Journal of Drug and Alcohol Abuse, 31(3), 455-469. https://doi.org /10.1081/ADA-200056807

Sharifi, V., Assadi, S. M., Mohammadi, M. R., Amini, H., Kaviani, H., Semnani, Y., Shabani, A., Shahrivar, Z., Davari-Ashtiani, R., Shooshtari, M. H., Seddigh, A., \& Jalali, M. (2009). A Persian translation of the Structured Clinical Interview for 
Diagnostic and Statistical Manual of Mental Disorders, Fourth Edition: Psychometric properties. Comprehensive Psychiatry, 50(1), $\quad 86-91 . \quad$ https://doi.org/10.1016 /j.comppsych.2008.04.004

Simkin, D. R., Thatcher, R. W., \& Lubar, J. (2014). Quantitative EEG and neurofeedback in children and adolescents: Anxiety disorders, depressive disorders, comorbid addition and attention-deficit/hyperactivity disorder, and brain injury. Child and Adolescent Psychiatric Clinics of North America, 23(3), 427-464. https://doi.org/10.1016/j.chc.2014.03.001

Ticci, C., Luongo, T., Valvo, G., Ferrari, A. R., Brovedani, P., Masi, G., Pellacani, S., \& Sicca, F. (2019). Clinical and electroencephalographic correlates of psychiatric features in children with frontal lobe epilepsy. Epilepsy \& Behavior, 92, 283-289. https://doi.org/10.1016/j.yebeh.2019.01.008
Vaschillo, E. G., Vaschillo, B., \& Lehrer, P. M. (2006). Characteristics of resonance in heart rate variability stimulated by biofeedback. Applied Psychophysiology and Biofeedback, 31(2), 129-142. https://doi.org/10.1007/s10484006-9009-3

Vernon, D., Egner, T., Cooper, N., Compton, T., Neilands, C., Sheri, A., \& Gruzelier, J. (2003). The effect of training distinct neurofeedback protocols on aspects of cognitive performance. International Journal of Psychophysiology, 47(1), 75-85. https://doi.org/10.1016/S0167-8760(02)00091-0

Received: May 10, 2021

Accepted: July 26, 2021

Published: September 30, 2021 